\title{
Jacobi-Eisenstein series of degree two over Cayley numbers
}

\author{
Minking Eie
}

\begin{abstract}
We shall develop the general theory of Jacobi forms of degree two over Cayley numbers and then construct a family of JacobiEisenstein series which forms the orthogonal complement of the vector space of Jacobi cusp forms of degree two over Cayley numbers. The construction is based on a group representation arising from the transformation formula of a set of theta series.
\end{abstract}

\section{Introduction and notations.}

The theory of Jacobi forms was first organized systematically in 1985 in the textbook by M. Eichler and D. Zagier (see [4]). In the book they provided a complete presentation of the readily known proof for the Saito-Kurokawa conjecture, which asserted the possible existence of a lifting from modular forms of degree one to Siegel modular forms of degree two. Analogous theories on various domains was substantially investigated with important results since then. Here we mention a few among them. This author and A. Krieg jointly initiated the theory of Jacobi forms on $\mathbf{H} \times \mathcal{C}_{\mathbb{C}}$ (see [5], [6], [7], [8]), the product space of upper half plane and Cayley numbers over the complex field $\mathbb{C}$, and he proved that there is an one-to-one correspondence between elliptic modular forms of weight $k-4$ and modular forms of weight $k$ belonging to the Maaß space on $\mathcal{H}_{2}$, the upper half plane of $2 \times 2$ Hermitian matrices over Cayley numbers. On the other hand, Ziegler (see [16]) 
considered Fourier-Jacobi expansions of Siegel modular forms and introduced Jacobi forms of several variables on $\mathbf{H}_{n} \times \mathbb{C}^{n m}$, the product space of Siegel upper half plane $\mathbf{H}_{n}$ and the vector space $\mathbb{C}^{n m}$ realized as $n \times m$ matrices over $\mathbb{C}$. A. Krieg also gave a general treatment for another kind of Jacobi forms of several variables (see [14]).

In 1993, Kim constructed a singular modular form of weight 4 on the 27 dimensional exceptional domain (see [13]) by the analytic continuation of a non-holomorphic Eisenstein series. Therefore it is duely important to investigate Jacobi forms on $\mathcal{H}_{2} \times \mathcal{C}_{\mathbb{C}}^{2}$ more thoroughly since they appear naturally to be Fourier-Jacobi coefficients of modular forms on the exceptional domain.

In this paper, we shall devolop the general theory of Jacobi forms on $\mathcal{H}_{2} \times \mathcal{C}_{\mathbb{C}}^{2}$. In particular, we are able to decompose a Jacobi form into an inner product of a vector-valued modular form and a vectorvalued theta series. By using the transformation formula of the vectorvalued theta series, we obtain a group representation $\psi$ of $\Gamma_{2}$, which is a discrete subgroup of the group of bi-holomorphic mappings from $\mathcal{H}_{2}$ onto itself. After doing so, we are finally able to construct a family of Jacobi-Eisenstein series by using this group representation $\psi$.

As an application, we shall determine the Fourier coefficients of singular modular forms of weight 4 and 8 explicitly, which paves a new way to construct singular modular forms on 27 dimensional exceptional domain via theory of Jacobi forms of degree two. The outcome of such construction is similar to the outcome proved by A. Krieg (see [16]), who applied the results in his joint work with Eie (see [6], [7]) to obtain an alternative proof that a function with a Fourier expansion obtained by $\mathrm{Kim}$ is indeed a modular form of weight 4 . This work provides a systemic and general approach to deal with the whole issue.

To describe Jacobi forms of degree two over Cayley numbers, we need notations concerning Cayley numbers as well as the modular group $\Gamma_{2}$.

Let $F$ be a field. The Cayley numbers $\mathcal{C}_{F}$ over $F$ is an eight dimensional vector space over $F$ with a standard basis $e_{0}, e_{1}, e_{2}, e_{3}, e_{4}, e_{5}, e_{6}$, $e_{7}$ satisfying the following rules of multiplication (see [1])

1) $x e_{0}=e_{0} x=x$ for all $x$ in $\mathcal{C}_{F}$,

2) $e_{j}^{2}=-e_{0}, j=1,2, \ldots, 7$, and

3) $e_{1} e_{2} e_{4}=e_{2} e_{3} e_{5}=e_{3} e_{4} e_{6}=e_{4} e_{5} e_{7}=e_{5} e_{6} e_{1}=e_{6} e_{7} e_{2}=$ $e_{7} e_{1} e_{3}=-e_{0}$. 
For $x=\sum_{j=0}^{7} x_{j} e_{j}$ and $y=\sum_{j=0}^{7} y_{j} e_{j}$ in $\mathcal{C}_{F}$, we define the following operations on $\mathcal{C}_{F}$.

1) Involution: $x \longrightarrow \bar{x}=x_{0} e_{0}-\sum_{j=1}^{7} x_{j} e_{j}$,

2) Trace operator: $T(x)=x+\bar{x}=2 x_{0}$,

3) Norm operator: $N(x)=x \bar{x}=\bar{x} x=\sum_{j=0}^{7} x_{j}^{2}$, and

4) Inner product: $\sigma: \mathcal{C}_{F} \times \mathcal{C}_{F} \longrightarrow F, \sigma(x, y)=T(x \bar{y})=T(y \bar{x})=$ $2 \sum_{j=0}^{7} x_{j} y_{j}$.

Note that we have the property: $N(x+y)=N(x)+N(y)+\sigma(x, y)$.

Denoted by $\mathbf{o}$ the $\mathbb{Z}$-module in $\mathcal{C}_{\mathbf{Q}}$, generated by $\alpha_{j}(j=0,1,2, \ldots$, 7) as follow

$$
\begin{array}{cc}
a_{0}=e_{0}, \quad \alpha_{1}=e_{1}, & a_{2}=e_{2}, \quad \alpha_{3}=-e_{4}, \\
\alpha_{4}=\frac{1}{2}\left(e_{1}+e_{2}+e_{3}-e_{4}\right), & \alpha_{5}=\frac{1}{2}\left(-e_{0}-e_{1}-e_{4}+e_{5}\right), \\
\alpha_{6}=\frac{1}{2}\left(-e_{0}+e_{1}-e_{2}+e_{6}\right), & \alpha_{7}=\frac{1}{2}\left(-e_{0}+e_{2}+e_{4}+e_{7}\right) .
\end{array}
$$

Elements in o are refered as integral Cayley numbers. This module o is denoted by $J$ in [3], it satisfies the following conditions:

1) $N(x) \in \mathbb{Z}$ and $T(x) \in \mathbb{Z}$ for each $x$ in the set,

2) the set is closed under addition and multiplication, and

3) the set contains $e_{0}$.

As shown there, o is maximal among those modules with these properties.

Let $\mathcal{H}_{2}$ denote the Hermitian upper half plane of degree two over Cayley numbers. More precisely, it is defined by the following

$$
\begin{aligned}
& \mathcal{H}_{2}=\left\{Z=\left[\begin{array}{cc}
x_{1} & x_{12} \\
\bar{x}_{12} & x_{2}
\end{array}\right]+i\left[\begin{array}{cc}
y_{1} & y_{12} \\
\bar{y}_{12} & y_{2}
\end{array}\right]:\right. \\
& \left.x_{1}, x_{2}, y_{1}, y_{2} \in \mathbb{R}, x_{12}, y_{12} \in \mathcal{C}_{\mathbb{R}}, y_{2}>0, y_{1} y_{2}-N\left(y_{12}\right)>0\right\} \text {. }
\end{aligned}
$$

Obviously, $\mathcal{H}_{2}$ is an open convex subdomain of $\mathbb{C}^{10}$. The domain $\mathcal{H}_{2}$ is isomorphic to the tube domain over the "light cone" of dimension 10 , 
the non-compact Hermitian space of $S O(10,2)$ (see [2, p. 71]). See also [5] or [6] for the details.

Given

$$
Z=\left[\begin{array}{cc}
z & w \\
\bar{w} & z^{*}
\end{array}\right] \in \mathcal{H}_{2},
$$

$Z$ is invertible and

$$
-Z^{-1}=\frac{-1}{z z^{*}-N(w)}\left[\begin{array}{cc}
z^{*} & -w \\
-\bar{w} & z
\end{array}\right] \in \mathcal{H}_{2} .
$$

Denoted by $\Gamma_{2}$ the discontinuous subgroup of the group of bi-holomorphic mappings from $\mathcal{H}_{2}$ onto itself, which is generated by the following:

1) $p_{B}: Z \longrightarrow Z+B, B=\left[\begin{array}{cc}n & t \\ \bar{t} & m\end{array}\right], n, m \in \mathbb{Z}, t \in \mathbf{o}$,

2) $t_{U}: Z \longrightarrow{ }^{t} \bar{U} Z U, U=\left[\begin{array}{cc}0 & 1 \\ -1 & 0\end{array}\right]$ or $\left[\begin{array}{ll}1 & t \\ 0 & 1\end{array}\right], t \in \mathbf{o}$, and

3) $\iota: Z \longrightarrow-Z^{-1}$.

We use $(T, Z)$ to denote the inner product of two Hermitian matrices $T$ and $Z ;(T, Z)=t_{1} z+t_{2} z^{*}+\sigma\left(t_{12}, w\right)$ if

$$
T=\left[\begin{array}{cc}
t_{1} & t_{12} \\
\bar{t}_{12} & t_{2}
\end{array}\right]
$$

and

$$
Z=\left[\begin{array}{cc}
z & w \\
\bar{w} & z^{*}
\end{array}\right]
$$

Also we let

$$
\Lambda_{2}=\left\{\left[\begin{array}{cc}
n & t \\
t & m
\end{array}\right]: n, m \in \mathbb{Z}, t \in \mathbf{o}\right\}
$$

\section{Jacobi forms of degree two over Cayley numbers.}

By a Jacobi form of degree two over Cayley numbers, we mean a Jacobi form defined on $\mathcal{H}_{2} \times \mathcal{C}_{\mathbb{C}}^{2}$. Let $k$ and $m \geq 1$ be non-negative integers. A holomorphic function $f: \mathcal{H}_{2} \times \mathcal{C}_{\mathbb{C}}^{2} \longrightarrow \mathbb{C}$ is called a Jacobi form of weight $k$ and index $m$ with respect to $\Gamma_{2}$ if $f$ satisfies the following conditions: 
J-1) $f(Z+B, W)=f(Z, W)$ for all $B \in \Lambda_{2}$, $t \in \mathbf{o}$,

J-2) $f\left(Z[U],{ }^{t} \bar{U} W\right)=f(Z, W)$ for all $U=\left[\begin{array}{ll}1 & 0 \\ t & 1\end{array}\right]$ or $\left[\begin{array}{cc}0 & 1 \\ -1 & 0\end{array}\right]$,

J-3) $f\left(-Z^{-1}, Z^{-1} W\right)=(\operatorname{det} Z)^{k} \exp \left(2 \pi i m Z^{-1}[W]\right) f(Z, W)$,

$$
\begin{aligned}
f(Z, W & +Z \mathbf{q}+\mathbf{p}) \\
& =\exp \left(-2 \pi i m\left(\left(Z, \mathbf{q}^{t} \overline{\mathbf{q}}\right)+\sigma\left(w_{1}, q_{1}\right)+\sigma\left(w_{2}, q_{2}\right)\right)\right) f(Z, W),
\end{aligned}
$$

for all $\mathbf{q}, \mathbf{p} \in \mathbf{o}^{2}$, and

J-5) $f$ has a Fourier expansion of the form

$$
f(Z, W)=\sum_{\mathbf{q} \in \mathbf{o}^{2}} \sum_{\substack{T \in \Lambda_{2} \\ T \geq \mathbf{q}^{t} \overline{\mathbf{q}} / m}} a(T, \mathbf{q}) e^{2 \pi i\left((T, Z)+\sigma\left(q_{1}, w_{1}\right)+\sigma\left(q_{2}, w_{2}\right)\right)} .
$$

Here for $Z=\left[\begin{array}{cc}z_{1} & z_{12} \\ \bar{z}_{12} & z_{2}\end{array}\right] \in \mathcal{H}_{2}$ and $W=\left[\begin{array}{c}w_{1} \\ w_{2}\end{array}\right] \in \mathcal{C}_{\mathbb{C}}^{2}$, we let

$$
Z[W]=z_{1} N\left(w_{1}\right)+z_{2} N\left(w_{2}\right)+\sigma\left(z_{12}, w_{1} \bar{w}_{2}\right) .
$$

For $2 \times 2$ Hermitian matrix $A=\left[\begin{array}{ll}a & \lambda \\ \lambda & b\end{array}\right]$, we write $A \geq 0$, to mean $a \geq 0, b \geq 0$ and $a b \geq N(\lambda)$. Also $A \geq B$ if and only if $A-B \geq 0$.

From the above definition, we are able to decompose a Jacobi form of degree two into an inner product of a vector-valued modular form and a vector-valued theta series.

Proposition 1. Let $f(Z, W)$ be a Jacobi form of degree two with Fourier expansion (2.1). Then

$$
f(Z, W)=\sum_{\mathbf{q}:(\mathbf{o} / m \mathbf{o})^{2}} F_{\mathbf{q}}(Z) \vartheta_{m, \mathbf{q}}(Z, W)
$$

with

$$
F_{\mathbf{q}}(Z)=\sum_{T \geq \mathbf{q}^{t} \overline{\mathbf{q}} / m} a(T, \mathbf{q}) e^{2 \pi i\left(T-\mathbf{q}^{t} \overline{\mathbf{q}} / m, Z\right)}
$$


and

$$
\vartheta_{m, \mathbf{q}}(Z, W)=\sum_{\substack{\mathbf{h}=\lambda+\mathbf{q} / m \\ \lambda \in \mathbf{o}^{2}}} \exp \left(2 \pi i m\left(\left(\mathbf{h}^{t} \overline{\mathbf{h}}, Z\right)+\sigma\left(h_{1}, w_{1}\right)+\sigma\left(h_{2}, w_{2}\right)\right)\right)
$$

Proof. Set $\mathbf{p}=\mathbf{q}+m \lambda$ with $\mathbf{q}$ ranges over all representatives of $(\mathbf{o} / m \mathbf{o})^{2}$ and $\lambda$ ranges over $\mathbf{o}^{2}$ in the first summation of $f(Z, W)$. Then our assertion follows form $(\mathrm{J}-4)$ and a direct verification.

For each $\mathbf{q}={ }^{t}\left(q_{1}, q_{2}\right) \in \mathbf{o}^{2}$, consider the theta series $\vartheta_{m, \mathbf{q}}(Z, W)$ defined by

$$
\begin{aligned}
\vartheta_{m, \mathbf{q}}(Z, W)=\sum_{\substack{\mathbf{h}=\lambda+\mathbf{q} / m \\
\lambda \in \mathbf{o}^{2}}} \exp \left(2 \pi i m \left(\left(\mathbf{h}^{t} \overline{\mathbf{h}}, Z\right)\right.\right. \\
\left.\left.+\sigma\left(h_{1}, w_{1}\right)+\sigma\left(h_{2}, w_{2}\right)\right)\right) .
\end{aligned}
$$

Obviously, one has

$$
\vartheta_{m, \mathbf{q}}(Z+B, W)=e^{2 \pi i\left(\mathbf{q}^{t} \overline{\mathbf{q}}, B\right) / m} \vartheta_{m, \mathbf{q}}(Z, W), \quad \text { for all } T \in \Lambda_{2},
$$

and

$$
\vartheta_{m, \mathbf{q}}\left(Z[U],{ }^{t} \bar{U} W\right)=\vartheta_{m, U \mathbf{q}}(Z, W),
$$

for $U=\left[\begin{array}{ll}1 & 0 \\ t & 1\end{array}\right]$ or $\left[\begin{array}{cc}0 & 1 \\ -1 & 0\end{array}\right], t \in \mathbf{o}$.

Here we shall prove the transformation formula between

$$
\vartheta_{m, \mathbf{q}}\left(-Z^{-1}, Z^{-1} W\right)
$$

and $\vartheta_{m, \mathbf{p}}(Z, W)$ for various $\mathbf{p}$ in $\mathbf{o}^{2}$. We need the following.

Lemma 1. For each $\mathbf{h}={ }^{t}\left(h_{1}, h_{2}\right) \in \mathcal{C}_{\mathbb{R}}^{2}, \Lambda=\operatorname{diag}\left[\xi_{1}, \xi_{2}\right], \xi_{1}>0$, $\xi_{2}>0$ one has

$$
\begin{array}{r}
\left(\mathbf{h}^{t} \overline{\mathbf{h}}, \Lambda[U]\right)=\left((U \mathbf{h})\left({ }^{t} \overline{\mathbf{h}}^{t} \bar{U}\right), \Lambda\right), \\
\text { for all } U=\left[\begin{array}{ll}
1 & t \\
0 & 1
\end{array}\right],\left[\begin{array}{ll}
1 & 0 \\
t & 1
\end{array}\right] \text { or }\left[\begin{array}{cc}
0 & 1 \\
-1 & 0
\end{array}\right], t \in \mathcal{C}_{\mathbb{R}}
\end{array}
$$


Proof. It is obvious for $U=\left[\begin{array}{cc}0 & 1 \\ -1 & 0\end{array}\right]$. Here we prove the case $U=\left[\begin{array}{ll}1 & t \\ 0 & 1\end{array}\right]$. We have

$$
\left(\mathbf{h}^{t} \overline{\mathbf{h}}, \Lambda[U]\right)=\xi_{1} N\left(h_{1}\right)+\xi_{2} \sigma\left(t, h_{1} \bar{h}_{2}\right)+\left(\xi_{2}+\xi_{1} N(t)\right) N\left(h_{2}\right) .
$$

On the other hand,

$$
U \mathbf{h}=\left[\begin{array}{c}
h_{1}+t h_{2} \\
h_{2}
\end{array}\right]
$$

It follows

$$
\begin{aligned}
\left((U \mathbf{h})\left({ }^{t} \overline{\mathbf{h}}^{t} \bar{U}\right), \Lambda\right) & =\xi_{1} N\left(h_{1}+t h_{2}\right)+\xi_{2} N\left(h_{2}\right) \\
& =\xi_{1} N\left(h_{1}\right)+\xi_{1} \sigma\left(h_{1}, t h_{2}\right)+\left(\xi_{1} N\left(t_{1}\right)+\xi_{2}\right) N\left(h_{2}\right) .
\end{aligned}
$$

Hence our assertion follows form the fact that

$$
\sigma\left(t, h_{1} \bar{h}_{2}\right)=T\left(t\left(h_{2} \bar{h}_{1}\right)\right)=T\left(\left(t h_{2}\right) \bar{h}_{1}\right)=\sigma\left(h_{1}, t h_{2}\right)
$$

In exactly the same way, we prove the following.

Lemma 2. For $\mathbf{h}={ }^{t}\left(h_{1}, h_{2}\right) \in \mathcal{C}_{\mathbb{R}}^{2}, \mathbf{V}={ }^{t}\left(v_{1}, v_{2}\right) \in \mathcal{C}_{\mathbb{R}}^{2}, \Lambda=$ $\operatorname{diag}\left[\xi_{1}, \xi_{2}\right], \xi_{1}>0, \xi_{2}>0$ and $U=\left[\begin{array}{ll}1 & t \\ 0 & 1\end{array}\right]$ or $\left[\begin{array}{ll}1 & 0 \\ t & 1\end{array}\right], t \in \mathcal{C}_{\mathbb{R}}$, one has

$$
\left.T\left({ }^{t} \overline{\mathbf{h}}\left(\Lambda^{-1}\left[{ }^{t} \bar{U}^{-1}\right]\right) \mathbf{V}\right)\right)=T\left(\left({ }^{t} \overline{\mathbf{h}} U^{-1}\right)\left(\Lambda^{-1}\left({ }^{t} \bar{U}^{-1} \mathbf{V}\right)\right) .\right.
$$

Lemma 3. For positive integer $m$ and $q \in \mathbf{o}$, let

$$
\vartheta_{m, q}(z, w)=\sum_{t \in \mathbf{o}} e^{2 \pi i m(N(t+q / m) z+\sigma(t+q / m), w))}, \quad(z, w) \in \mathbf{H} \times \mathcal{C}_{\mathbb{R}}
$$

Then for $y>0, v \in \mathcal{C}_{\mathbb{R}}$

$$
\vartheta_{m, q}\left(i y^{-1}, \frac{v}{y}\right)=e^{-2 \pi m N(v) / y}\left(\frac{y}{m}\right)^{4} \sum_{t \in \mathbf{o}} e^{-2 \pi y N(t) / m} e^{-2 \pi i \sigma(q / m-i v, t)} .
$$


Proof. Let $S$ be the matrix corresponding to the quadratic form in $g_{j}(j=0,1, \ldots, 7)$ of $2 N\left(\sum_{j=0}^{7} g_{j} \alpha_{j}\right)$, i.e., $S=\left(\sigma\left(\alpha_{i}, \alpha_{j}\right)\right)_{0 \leq i, j \leq 7}$ and $\widehat{q}, \widehat{v}$ be the representatives of $q, v$ with the basis $\alpha_{0}, \alpha_{1}, \ldots, \alpha_{7}$. Then

$$
\begin{aligned}
\vartheta_{m, q}\left(i y^{-1}, \frac{v}{y}\right) & =\sum_{t \in \mathbf{o}} e^{-2 \pi m\left[N(t+q / m) y^{-1}+\sigma(t+q / m,-i v / y)\right]} \\
& =e^{-2 \pi m N(v) / y} \sum_{\widehat{t} \in \mathbb{Z}^{8}} e^{-\pi m S[\widehat{t}+\widehat{q} / m-i \widehat{v}] y^{-1}} .
\end{aligned}
$$

$S$ is a positive definite symmetric integral matrix with $\operatorname{det} S=1$. By a direct calculation, the Fourier transform of the function

$$
f(x)=e^{-\pi m S[x+\widehat{q} / m-i \widehat{v}] y^{-1}}
$$

is given by

$$
\widehat{f}(\eta)=\left(\frac{y}{m}\right)^{4} e^{-2 \pi i(\widehat{q} / m-i \widehat{v}, \eta)} e^{-\pi y S^{-1}[\eta] / m} .
$$

Here $\langle\alpha, \beta\rangle$ is the usual inner product of $\alpha, \beta$ in $\mathbb{R}^{8}$. Then the Poisson summation formula implies that

$$
\begin{aligned}
\vartheta_{m, q}\left(i y^{-1}, \frac{v}{y}\right) & =e^{-2 \pi m N(v) / y}\left(\frac{y}{m}\right)^{4} \sum_{\eta \in \mathbb{Z}^{8}} e^{-2 \pi i(\widehat{q} / m-i \widehat{v}, y)} e^{-\pi y S^{-1}[\eta] / m} \\
& =e^{-2 \pi m N(v) / y}\left(\frac{y}{m}\right)^{4} \sum_{t \in \mathbf{o}} e^{-2 \pi y N(t) / m} e^{-2 \pi i \sigma(q / m-i v, t)}
\end{aligned}
$$

Proposition 2. Suppose that $\vartheta_{m, \mathbf{q}}(Z, W)$ is defined as in (2.2). Then

$$
\vartheta_{m, \mathbf{q}}\left(-Z^{-1}, Z^{-1} W\right)
$$

$$
\begin{aligned}
= & (\operatorname{det} Z)^{4} \exp \left(2 \pi i m Z^{-1}[W]\right) \\
& \cdot \frac{1}{m^{8}} \sum_{\mathbf{p}:(\mathbf{o} / m \mathbf{o})^{2}} e^{-2 \pi i\left[\sigma\left(q_{1}, p_{1}\right)+\sigma\left(q_{2}, p_{2}\right)\right] / m} \vartheta_{m, \mathbf{p}}(Z, W) .
\end{aligned}
$$

Proof. It suffices to prove that (2-5) holds for $Z=i Y$ and $W=i V$ since both sides are holomorphic functions in $Z$ and $W$. Let $Y=\Lambda[U]$ with $\Lambda=\operatorname{diag}\left[\xi_{1}, \xi_{2}\right]$ and $U=\left[\begin{array}{ll}1 & t \\ 0 & 1\end{array}\right]$. Then

$$
Y^{-1}=\Lambda^{-1}\left[{ }^{t} U^{-1}\right]
$$


It follows from lemmas 1 and 2 that

$$
\begin{gathered}
\vartheta_{m, \mathbf{q}}\left(i Y^{-1}, Y^{-1} V\right) \\
=\sum_{\substack{\mathbf{h}=\lambda+\mathbf{q} / m \\
\lambda \in \mathbf{o}^{2}}} \exp \left(-2 \pi m\left(\mathbf{h}^{t} \overline{\mathbf{h}}, Y^{-1}\right)+2 \pi i m T\left({ }^{t} \overline{\mathbf{h}}\left(Y^{-1} V\right)\right)\right) \\
=\sum_{\substack{\mathbf{h}=\lambda+\mathbf{q} / m \\
\lambda \in \mathbf{o}^{2}}} \exp \left(-2 \pi m\left(\mathbf{h}^{t} \overline{\mathbf{h}}, \Lambda^{-1}\left[{ }^{t} U^{-1}\right]\right)\right. \\
\left.\quad+2 \pi i m T\left(\left({ }^{t} \overline{\mathbf{h}}\right)\left(\Lambda^{-1}\left[{ }^{t} \bar{U}^{-1}\right] V\right)\right)\right) \\
\sum_{\substack{\mathbf{h}=\lambda+\mathbf{q} / m \\
\lambda \in \mathbf{o}^{2}}} \exp \left(-2 \pi m\left(\left({ }^{t} U^{-1} \mathbf{h}\right)\left({ }^{t} \overline{\mathbf{h}} U^{-1}\right), \Lambda^{-1}\right)\right. \\
\end{gathered}
$$

Note that by Lemma 3, we have

$$
\begin{aligned}
& \sum_{\substack{\mathbf{h}=\lambda+\mathbf{q} / m \\
\lambda \in \mathbf{o}^{2}}} \exp \left(-2 \pi m\left(h^{t} \bar{h}, \Lambda^{-1}\right)+2 \pi i m T\left({ }^{t} \bar{h}\left(\Lambda^{-1} V\right)\right)\right) \\
= & \prod_{j=1}^{2} \sum_{t_{j} \in \mathbf{o}} \exp \left(-2 \pi m N\left(t_{j}+\frac{q_{j}}{m}\right) \xi_{j}^{-1}+2 \pi i \sigma\left(t_{j}+\frac{q_{j}}{m}, \frac{v_{j}}{\xi_{j}}\right)\right) \\
= & \prod_{j=1}^{2}\left(\frac{\xi_{j}}{m}\right)^{4} e^{-2 \pi m} N\left(v_{j}\right) \xi_{j}^{-1} \sum_{h_{j} \in \mathbf{o}} e^{-2 \pi \xi_{j} N\left(h_{j}\right) / m} e^{-2 \pi i \sigma\left(q_{j} / m-i v_{j}, h_{j}\right)} \\
= & \frac{\left(\xi_{1} \xi_{2}\right)^{4}}{m^{8}} e^{-2 \pi m \Lambda^{-1}[V]} \sum_{\mathbf{h} \in \mathbf{o}^{2}} e^{-2 \pi m^{-1}\left(h^{t} \bar{h}, \Lambda\right)} e^{\left.-2 \pi i T\left({ }^{t} \bar{h}\right)(q / m-i V)\right)} .
\end{aligned}
$$

With a linear transformation $\mathbf{h} \longrightarrow{ }^{t} U^{-1} \mathbf{h}$, it causes a change in the Fourier transform of the corresponding function and yields

$$
\begin{aligned}
=\sum_{\substack{\mathbf{h}=\lambda+\mathbf{q} / m \\
\lambda \in \mathbf{o}^{2}}} \exp ( & -2 \pi m\left(\left({ }^{t} U^{-1} h\right)\left({ }^{t} \bar{h} U^{-1}\right), \Lambda^{-1}\right) \\
& \left.\quad+2 \pi i m T\left(\left({ }^{t} \overline{\mathbf{h}} U^{-1}\right) \Lambda^{-1}\left({ }^{t} \bar{U}^{-1} V\right)\right)\right)
\end{aligned}
$$




$$
\begin{aligned}
= & e^{-2 \pi m \Lambda^{-1}\left[\bar{U}^{-1} V\right]}\left(\xi_{1} \xi_{2}\right)^{4} m^{-8} \\
& \cdot \sum_{\mathbf{h} \in \mathbf{o}^{2}} \exp \left(-2 \pi m^{-1}\left((U h)\left({ }^{t} \bar{h}^{t} \bar{U}\right), \Lambda\right)\right. \\
& \left.\quad-2 \pi i T\left({ }^{t} \bar{h}^{t} \bar{U}\right)\left({ }^{t} \bar{U}^{-1}\right)\left(\frac{q}{m}-i V\right)\right) \\
= & e^{-2 \pi m Y^{-1}[V]}(\operatorname{det} Y)^{4} m^{-8} \\
& \cdot \sum_{\mathbf{h} \in \mathbf{o}^{2}} \exp \left(-2 \pi m^{-1}\left(h^{t} \bar{h}, Y\right)\right. \\
& \left.\quad-2 \pi i \sigma\left(\frac{q_{1}}{m}-i v_{1}, h_{1}\right)-2 \pi i \sigma\left(\frac{q_{2}}{m}-i v_{2}, h_{2}\right)\right) .
\end{aligned}
$$

Now set $\mathbf{h}=\mathbf{p}+m \lambda$ with $\mathbf{p}$ ranges over a set of representatives of $\mathbf{o} / m \mathbf{o}$ and $\lambda$ ranges over $\mathbf{o}^{2}$, the above is equal to. This proves our assertion.

We then have the following result concerning a group representation of $\Gamma_{2}$.

Proposition 3. There exists a group homomorphism $\psi: \Gamma_{2} \longrightarrow$ $U\left(m^{16}\right)$, unitary group of size $m^{16}$, determined by

A) $\psi\left(p_{B}\right)=\operatorname{diag}\left[e^{-2 \pi i\left(\mathbf{q}^{t} \overline{\mathbf{q}}, B\right)}\right]_{\mathbf{q}:(\mathbf{o} / m \mathbf{o})^{2}}, B \in \Lambda_{2}$,

B) $\psi\left(t_{U}\right)=\left[s_{\mathbf{p}, \mathbf{q}}\right]_{\mathbf{p}, \mathbf{q}:(\mathbf{o} / m \mathbf{o})^{2}}$ with

$$
s_{\mathbf{p}, \mathbf{q}}= \begin{cases}1, & \text { if } \mathbf{q}=U \mathbf{p}, \\ 0, & \text { otherwise },\end{cases}
$$

for $U=\left[\begin{array}{ll}1 & t \\ 0 & 1\end{array}\right]$ or $\left[\begin{array}{cc}0 & 1 \\ -1 & 0\end{array}\right], t \in \mathbf{o}$, and

C) $\psi(\iota)=\frac{1}{m^{8}}\left(e^{2 \pi i\left[\sigma\left(p_{1}, q_{1}\right)+\sigma\left(p_{2}, q_{2}\right)\right] / m}\right)_{\mathbf{p}, \mathbf{q}:(\mathbf{o} / m \mathbf{o})^{2}}$.

Proof. $\psi$ is wholy determined by its images on the generators $p_{B}, t_{U}$ and $\iota$ of $\Gamma_{2}$. Fix a set of representatives

$$
q_{1}, q_{2}, \ldots, q_{m^{16}}
$$

of $(\mathbf{o} / m \mathbf{o})^{2}$ and let

$$
\Theta(Z, W)={ }^{t}\left(\vartheta_{m, q_{1}}(Z, W), \ldots, \vartheta_{m, q_{m} 16}(Z, W)\right) .
$$


As functions of $W$, the set of theta functions $\vartheta_{m, q_{j}}(Z, W)(j=1,2$, $\ldots, m)$ have different Fourier expansions. Each Fourier coefficient of $\vartheta_{m, q_{j}}(Z, W)$ is a function of $Z$ which depends, in particular, on the corresponding $q_{j}$. Therefore different Fourier coefficients for different $q_{j}$ are linear independent (as functions of $W$ ). We conclude that the set of theta functions $\vartheta_{m, q_{j}}(Z, W)$ are linearly independent.

Form $(2.3),(2.4)$ and $(2.5)$, we let

$$
\begin{gathered}
\Theta(Z+B, W)=\overline{\psi\left(p_{B}\right)} \Theta(Z, W), \\
\Theta\left(Z[U],{ }^{t} \bar{U} W\right)=\overline{\psi\left(t_{U}\right)} \Theta(Z, W),
\end{gathered}
$$

and

$$
\Theta\left(-Z^{-1}, Z^{-1} W\right)=(\operatorname{det} Z)^{4} \exp \left(2 \pi i m Z^{-1}[W]\right) \overline{\psi(\iota)} \Theta(Z, W) .
$$

Then $\psi$ has the properties A), B) and C) as asserted.

\section{Jacobi-Eisenstein series.}

As shown in Proposition 1, we are able to decompose a Jacobi form of degree two into an inner product of a vector-valued modular form and a vector-valued theta series. Now with the properties (2.3), (2.4), (2.5) of the theta series defined in (2.2), we can characterize a Jacobi form as a vector-valued modular form.

Proposition 4. Let $\mathbf{q}_{1}, \mathbf{q}_{2}, \ldots, \mathbf{q}_{m^{16}}$ be a set of representatives of $(\mathbf{o} / m \mathbf{o})^{2}$ and

$$
\begin{gathered}
F(Z)={ }^{t}\left(F_{\mathbf{q}_{1}}(Z), F_{\mathbf{q}_{2}}(Z), \ldots, F_{\mathbf{q}_{m^{16}}}(Z)\right), \\
\Theta(Z, W)={ }^{t}\left(\vartheta_{m, \mathbf{q}_{1}}(Z, W), \ldots, \vartheta_{m, \mathbf{q}_{m} 16}(Z, W)\right),
\end{gathered}
$$

with $\vartheta_{m, \mathbf{q}}(Z, W)$ as defined in (2.2) and

$$
F_{\mathbf{q}}(Z)=\sum_{\substack{T \in \Lambda_{2} \\ T \geq \mathbf{q}^{t} \overline{\mathbf{q}} / m}} \alpha(T, \mathbf{q}) e^{2 \pi i\left(T-\mathbf{q}^{t} \overline{\mathbf{q}} / m, Z\right)} .
$$

Then the following statements are equivalent: 
A) $f(Z, W)={ }^{t} F(Z) \cdot \Theta(Z, W)$ is a Jacobi form of weight $k$ and index $m$ with respect to $\Gamma_{2}$.

B) $F(Z)$ satisfies the following conditions:

1) $F(Z+B)=\psi\left(p_{B}\right) F(Z)$ for $B \in \Lambda_{2}$,

and

2) $F(Z[U])=\psi\left(t_{U}\right) F(Z)$ for $U=\left[\begin{array}{ll}1 & t \\ 0 & 1\end{array}\right]$ or $\left[\begin{array}{cc}0 & 1 \\ -1 & 0\end{array}\right], t \in \mathbf{o}$,

3) $F\left(-Z^{-1}\right)=(\operatorname{det} Z)^{k-4} \psi(\iota) F(Z)$.

Proof. It is a direct consequence of (2.3), (2.4) and Proposition 3.

Corollary. For positive integer $k \geq 4$, the correspondence

$$
F(Z) \longrightarrow F(Z) \vartheta_{1, \mathbf{0}}(Z, W)
$$

establishes an one to one correspondence between modular forms of weight $k-4$ on $\mathcal{H}_{2}$ and Jacobi forms of weight $k$ and index 1 on $\mathcal{H}_{2} \times \mathcal{C}_{\mathbb{C}}^{2}$.

Now we use the group homomorphism $\psi$ to construct a vectorvalued modular form corresponding to a Jacobi form of degree two. Let $j(g, Z)$ be a factor of the determinant of Jacobian matrix of $g \in \Gamma_{2}$ at $Z \in \mathcal{H}_{2}$ determined by the following:

1) $j\left(p_{B}, Z\right)=1$ for all $B \in \Lambda_{2}$,

2) $j\left(t_{U}, Z\right)=1$ for all $U=\left[\begin{array}{ll}1 & t \\ 0 & 1\end{array}\right]$ or $\left[\begin{array}{cc}0 & 1 \\ -1 & 0\end{array}\right], t \in \mathbf{o}$,

3) $j(\iota, Z)=\operatorname{det}(-Z)$, and

4) $j\left(g_{1} g_{2}, Z\right)=j\left(g_{1}, g_{2}(Z)\right) j\left(g_{2}, Z\right)$.

Also let $\Gamma_{2}^{0}$ be the subgroup of $\Gamma_{2}$ generated by $p_{B}$ and $t_{U}, B \in \Lambda_{2}$, $U=\left[\begin{array}{ll}1 & t \\ 0 & 1\end{array}\right]$ or $\left[\begin{array}{cc}0 & 1 \\ -1 & 0\end{array}\right], t \in \mathbf{o}$. For each $\mathbf{q} \in \mathbf{o}^{2}$ with $\mathbf{q}^{t} \overline{\mathbf{q}} \equiv 0$ $(\bmod m)$, we define

$$
\begin{aligned}
& E_{k, m}(Z, W ; \mathbf{q}) \\
& \quad={ }^{t}\left(E_{\mathbf{q}, \mathbf{q}_{1}}(Z), E_{\mathbf{q}, \mathbf{q}_{2}}(Z), \ldots, E_{\mathbf{q}, \mathbf{q}_{m} 16}(Z)\right) \cdot \Theta(Z, W)
\end{aligned}
$$


with

$$
E_{\mathbf{q}, \mathbf{p}}(Z)=\sum_{M: \Gamma_{2} / \Gamma_{2}^{0}} j(M, Z)^{4-k} \overline{\psi_{\mathbf{q}, \mathbf{p}}(M)}
$$

where

$$
\psi(M)=\left(\psi_{\mathbf{q}, \mathbf{p}}(M)\right)_{\mathbf{q}, \mathbf{p}:(\mathbf{o} / m \mathbf{o})^{2}} .
$$

The series in (3.4) converges absolutely and uniformly on compact subsets of $\mathcal{H}_{2}$ if $k>22$. Here we shall prove that the vector-valued modular form corresponding to $E_{k, m}(Z, W ; \mathbf{q})$ satisfies condition B) of Proposition 4. Consequently, $E_{k, m}(Z, W ; \mathbf{q})$ is indeed a Jacobi form of weight $k$ and index $m$ for $k>22$ and $\mathbf{q}^{t} \overline{\mathbf{q}} \equiv 0(\bmod m)$.

Proposition 5. For $k>22$ and $\mathbf{q} \in \mathbf{o}^{2}$ with $\mathbf{q}^{t} \overline{\mathbf{q}} \equiv 0(\bmod m)$, the Jacobi-Eisenstein defined in (3.3) and (3.4) is a Jacobi form of weight $k$ and index $m$.

Proof. Let $E(Z ; \mathbf{q})$ be the vector-valued modular form corresponding to $E_{k, m}(Z, W ; \mathbf{q})$. Then ${ }^{t} E(Z ; \mathbf{q})$ is the $\mathbf{q}$-th row of the matrix function defined by

$$
G(Z)=\sum_{M: \Gamma_{2} / \Gamma_{2}^{0}} j(M, Z)^{4-k} \overline{\psi(M)}
$$

Note that

$$
\psi\left(p_{B} M\right)=\psi\left(p_{B}\right) \psi(M)
$$

with

$$
\psi\left(p_{B}\right)=\operatorname{diag}\left[e^{-2 \pi i\left(\mathbf{q}^{t} \mathbf{q}, B\right) / m}\right]_{\mathbf{q}:(\mathbf{o} / m \mathbf{o})^{2}} .
$$

Thus the function $G$ may depend on the choice of coset representatives of $\Gamma_{2} / \Gamma_{2}^{0}$. However, its $\mathbf{q}-$ th row is independent of the choice since $\mathbf{q}^{t} \overline{\mathbf{q}} \equiv 0(\bmod m)$ and

$$
e^{-2 \pi i\left(\mathbf{q}^{t} \overline{\mathbf{q}}, B\right) / m}=1, \quad \text { for all } B \in \Lambda_{2} .
$$

From the group properties of $\Gamma_{2}$ and the cocycle condition of $j$,

$$
j(M, K(Z))=j(M K, Z) j(K, Z)^{-1}, \quad M, K \in \Gamma_{2},
$$

we conclude that

$$
{ }^{t} E(K(Z) ; \mathbf{q})=j(K, Z)^{k-4 t} E(Z ; \mathbf{q}) \overline{\psi\left(K^{-1}\right)},
$$


for all $K \in \Gamma_{2}$. Since $\psi(K)$ is unitary, it follows that

$$
E(K(Z) ; \mathbf{q})=j(K, Z)^{k-4} \psi(K) E(Z ; \mathbf{q}) \text {. }
$$

\section{Jacobi cusp forms.}

A Jacobi form $f$ of weight $k$ and index $m$ with the Fourier expansion

$$
f(Z, W)=\sum_{T \geq \mathbf{q}^{t} \mathbf{q} / m} \sum_{\mathbf{q} \in(\mathbf{o} / m \mathbf{o})^{2}} a(T, \mathbf{q}) e^{2 \pi i\left[(T, Z)+\sigma\left(q_{1}, w_{1}\right)+\sigma\left(q_{2}, w_{2}\right)\right]}
$$

is called a Jacobi cusp form if it satisfies the further condition

$$
a(T, \mathbf{q})=0, \quad \text { if } \operatorname{det}\left(T-\frac{1}{m} \mathbf{q}^{t} \overline{\mathbf{q}}\right)=0 .
$$

Let ${ }^{t}\left(F_{\mathbf{q}}(Z)\right)_{\mathbf{q}:(\mathbf{o} / m \mathbf{o})^{2}}$ and ${ }^{t}\left(G_{\mathbf{q}}(Z)\right)_{\mathbf{q}:(\mathbf{o} / m \mathbf{o})^{2}}$ be vector-valued modular forms corresponding to Jacobi forms $f$ and $g$ of weight $k$ and index $m$, respectively. For integer $k>22$, when at least one of $f$ and $g$ is a Jacobi cusp form, we define the inner product of $f$ and $g$ by

$$
\langle f, g\rangle=\int_{\Gamma_{2} \backslash \mathcal{H}_{2}}(\operatorname{det} Y)^{k-14} \sum_{\mathbf{q}:(\mathbf{o} / m \mathbf{o})^{2}} F_{\mathbf{q}}(Z) \overline{G_{\mathbf{q}}(Z)} d X d Y,
$$

where $\Gamma_{2} \backslash \mathcal{H}_{2}$ is a fundamental domain of $\mathcal{H}_{2}$ under the action of $\Gamma_{2}$ and $d X d Y$ is the usual Euclidean measure on $\mathcal{H}_{2}$

$$
\begin{gathered}
d X d Y=d x_{1} d x_{2} d x_{12} d y_{1} d y_{2} d y_{12} \\
Z=\left[\begin{array}{cc}
x_{1} & x_{12} \\
\bar{x}_{12} & x_{2}
\end{array}\right]+i\left[\begin{array}{cc}
y_{1} & y_{12} \\
\bar{y}_{12} & y_{2}
\end{array}\right] .
\end{gathered}
$$

Note that $(\operatorname{det} Y)^{-10} d X d Y$ is an invariant measure on $\mathcal{H}_{2}$.

With the inner product as given in (4.2), a Jacobi cusp form is orthogonal to a Jacobi-Eisenstein series of the same weight and index.

Proposition 6. Let $f$ be a Jacobi cusp form of weight $k$ and index $m$ and $E_{k, m}(Z, W ; \mathbf{q})$ be the Jacobi-Eisenstein series defined in (3.3) for $\mathbf{q}^{t} \overline{\mathbf{q}} \equiv 0(\bmod m)$. Then for $k>22$,

$$
\left\langle E_{k, m}, f\right\rangle=0 \text {. }
$$


Proof. Let

$$
{ }^{t}\left(F_{\mathbf{q}}(Z)\right) \mathbf{q}:(\mathbf{o} / m \mathbf{o})^{2}
$$

be the vector-valued modular form of $f$ and $\alpha(T, \mathbf{q})$ be the Fourier coefficient of $f$. Then we have

$$
F_{\mathbf{q}}(Z)=\sum_{T>\mathbf{q}^{t} \overline{\mathbf{q}} / m} \alpha(T, \mathbf{q}) e^{2 \pi i\left(T-\mathbf{q}^{t} \overline{\mathbf{q}} / m, Z\right)} .
$$

For $\mathbf{q}^{t} \overline{\mathbf{q}} \equiv 0(\bmod m)$, we have $\mathbf{q}^{t} \overline{\mathbf{q}} / m \in \Lambda_{2}$ and for all $T>\mathbf{q}^{t} \overline{\mathbf{q}} / m$

$$
\int_{\mathcal{J}_{\mathbb{R}}^{(2)} / \Lambda_{2}} e^{-2 \pi i\left(T-\mathbf{q}^{t} \overline{\mathbf{q}} / m, X\right)} d X=0,
$$

where $\mathcal{J}_{\mathbb{R}}^{(2)}$ is the set of $2 \times 2$ Hermitian matrices over real Cayley numbers. Thus it follows

$$
\int_{\Gamma_{2}^{0} \backslash \mathcal{H}_{2}}(\operatorname{det} Y)^{k-14} \overline{F_{\mathbf{q}}(Z)} d X d Y=0
$$

since we are able to construct a fundamental domain with its real part given by $\mathcal{J}_{\mathbb{R}}^{(2)} / \Lambda_{2}$ on $\mathcal{H}_{2}$ for $\Gamma_{2}^{0}$. Hence

$$
\int_{\Gamma_{2} \backslash \mathcal{H}_{2}}(\operatorname{det} Y)^{k-14} \sum_{M: \Gamma_{2} / \Gamma_{2}^{0}}|j(M, Z)|^{8-2 k} \overline{F_{\mathbf{q}}(M(Z))} d X d Y=0 .
$$

In light of the formula

$$
F_{\mathbf{q}}(M(Z))=\sum_{\mathbf{p}:(\mathbf{o} / m \mathbf{o})^{2}} j(M, Z)^{k-4} \psi_{\mathbf{q}, \mathbf{p}}(M) F_{\mathbf{p}}(Z),
$$

we get our assertion.

Next we shall count the number of different Jacobi-Eisenstein series defined in (3.3). For $m=p^{\nu}$, by the proof of [10, Proposition 5], we have

$$
{ }^{\#}\left\{\mathbf{q} \in(\mathbf{o} / m \mathbf{o})^{2}: \mathbf{q}^{t} \overline{\mathbf{q}} \equiv 0(\bmod m)\right\}
$$

$$
=p^{8 \nu}\left(\sum_{\tau=0}^{\nu} p^{3 \tau}-\sum_{\tau=0}^{\nu-1} p^{3 \tau-5}\right) .
$$


Set

$$
\begin{array}{r}
N_{m}={ }^{\#}\left\{\mathbf{q} \in(\mathbf{o} / m \mathbf{o})^{2}:\right. \\
\left.\mathbf{q}^{t} \overline{\mathbf{q}} \equiv 0(\bmod m)\right), \\
2 \mathbf{q} \equiv 0(\bmod m)\} .
\end{array}
$$

By (4.3) and an elementary consideration yield

$$
N_{m}= \begin{cases}1, & \text { if } m \equiv 1(\bmod 2) \\ 2240, & \text { if } m \equiv 2(\bmod 4) \\ 65536, & \text { if } m \equiv 0(\bmod 4)\end{cases}
$$

Now fix a particular set of representatives of $(\mathbf{o} / m \mathbf{o})^{2}$ as follow

$$
\begin{gathered}
\mathbf{q}_{1}, \ldots, \mathbf{q}_{r}, \mathbf{q}_{r+1}, \ldots, \mathbf{q}_{r+s},-\mathbf{q}_{r+1}, \ldots,-\mathbf{q}_{r+s}, \\
r=N_{m}, r+2 s=m^{16}
\end{gathered}
$$

with $2 \mathbf{q}_{j} \equiv 0(\bmod m)$ for $1 \leq j \leq r$ and $2 \mathbf{q}_{l} \not \equiv 0(\bmod m)$ for $r+1 \leq l \leq r+s$. With the above as an index set of $(\mathbf{o} / m \mathbf{o})^{2}$, we have the following

Proposition 7. Let $\iota$ be the transform $Z \longrightarrow-Z^{-1}$. Then

$$
\psi\left(\iota^{2}\right)=\left[\begin{array}{ccc}
E_{r} & 0 & 0 \\
0 & 0 & E_{s} \\
0 & E_{s} & 0
\end{array}\right] .
$$

where $E_{l}$ is the identity matrix of size $l \times l$.

Proof. Let $S_{\mathbf{p}, \mathbf{q}}$ be the entry of $\psi\left(\iota^{2}\right)=\psi(\iota) \psi(\iota)$ at $(\mathbf{p}, \mathbf{q})$ position. Then

$$
\begin{aligned}
S_{\mathbf{p}, \mathbf{q}} & =\frac{1}{m^{16}} \sum_{\mathbf{r}:(\mathbf{o} / m \mathbf{o})^{2}} e^{2 \pi i\left[\sigma\left(p_{1}, r_{1}\right)+\sigma\left(p_{2}, r_{2}\right)\right] / m} e^{2 \pi i\left[\sigma\left(r_{1}, q_{1}\right)+\sigma\left(r_{2}, q_{2}\right)\right] / m} \\
& =\frac{1}{m^{16}} \sum_{r_{1}: \mathbf{o} / m \mathbf{o}} e^{2 \pi i \sigma\left(p_{1}+q_{1}, r_{1}\right) / m} \sum_{r_{2}: \mathbf{o} / m \mathbf{o}} e^{2 \pi i \sigma\left(p_{2}+q_{2}, r_{2}\right) / m} \\
& = \begin{cases}1, & \text { if } p_{1}+q_{1} \equiv 0(\bmod m) \text { and } p_{2}+q_{2} \equiv 0(\bmod m), \\
0, & \text { otherwise }\end{cases}
\end{aligned}
$$


Thus our assertion follows.

Proposition 8. For any $M \in \Gamma_{2}, \psi(M)$ appears to be the form

$$
\left[\begin{array}{ccc}
A_{r} & H_{r s} & H_{r s} \\
F_{s r} & B_{s} & G_{s} \\
F_{s r} & G_{s} & B_{s}
\end{array}\right] .
$$

where $A_{r}$ is a matrix of size $r \times r, B_{s}, G_{s}$, are matrices of size $s \times s, H_{r s}$ is a matrix of size $r \times s$ and $F_{s r}$ is a matrix of size $s \times r$. In particular, one has

$$
\psi_{-\mathbf{q}, \mathbf{p}}(M)=\psi_{\mathbf{q},-\mathbf{p}}(M)
$$

Proof. It follows from $\psi(M)$ commutes with $\psi\left(\iota^{2}\right)=\psi\left(t_{U}\right)$ with $U=-E_{2}$.

Proposition 9. For any $\mathbf{q} \in \mathbf{o}^{2}$ with $\mathbf{q}^{t} \overline{\mathbf{q}} \equiv 0(\bmod m)$, one has

$$
E_{k, m}(Z, W ;-\mathbf{q})=E_{k, m}(Z, W ; \mathbf{q}) \text {. }
$$

PROOF.

$$
\begin{aligned}
E_{k, m}(Z, W ;-\mathbf{q}) & =\sum_{M: \Gamma_{2} \backslash \Gamma_{2}^{0}} j(M, Z)^{4-k} \sum_{\mathbf{p}:(\mathbf{o} / m \mathbf{o})^{2}} \overline{\psi_{-\mathbf{q}, \mathbf{p}}(M)} \vartheta_{m, \mathbf{p}}(Z, W) \\
& =\sum_{M: \Gamma_{2} \backslash \Gamma_{2}^{0}} j(M, Z)^{4-k} \sum_{\mathbf{p}:(\mathbf{o} / m \mathbf{o})^{2}} \overline{\psi_{\mathbf{q},-\mathbf{p}}(M)} \vartheta_{m, \mathbf{p}}(Z, W) \\
& =\sum_{M: \Gamma_{2} \backslash \Gamma_{2}^{0}} j(M, Z)^{4-k} \sum_{\mathbf{p}:(\mathbf{o} / m \mathbf{o})^{2}} \overline{\psi_{\mathbf{q}, \mathbf{p}}(M)} \vartheta_{m,-\mathbf{p}}(Z, W) \\
& =\sum_{M: \Gamma_{2} \backslash \Gamma_{2}^{0}} j(M, Z)^{4-k} \sum_{\mathbf{p}:(\mathbf{o} / m \mathbf{o})^{2}} \overline{\psi_{\mathbf{q}, \mathbf{p}}(M)} \vartheta_{m, \mathbf{p}}(Z,-W) \\
& =E_{k, m}(Z,-W ; \mathbf{q}) \\
& =E_{k, m}\left(Z\left[-E_{2}\right],\left(-E_{2}\right) W ; \mathbf{q}\right) \\
& =E_{k, m}(Z, W ; \mathbf{q}) .
\end{aligned}
$$

By (4.3), (4.5) and the previous proposition, we then have 
Proposition 10. The total number of different Jacobi-Eisenstein series is given by

$$
\frac{1}{2}\left(m^{8} \prod_{p \mid m}\left(\sum_{\tau=0}^{\nu_{p}(m)} p^{3 \tau}-\sum_{\tau=0}^{\nu_{p}(m)-1} p^{3 \tau-5}\right)+N_{m}\right)
$$

where $\nu_{p}(m)$ is the valuation defined by $\nu_{p}(m)=\alpha$ if $p^{\alpha}$ is the highest power of $p$ dividing $m$.

The set of Jacobi-Eisentein series is the orthogonal complement of the vector space of Jacobi cusp form which is denoted by $J_{k, m}^{0}\left(\Gamma_{2}\right)$ with respect to the inner product (4.2). By realizing Jacobi cusp forms as vector-valued cusp forms, we are able to compute its dimension via Selberg trace formula as we had done in [10, Appendix]: For $k>22$,

$$
\begin{gathered}
\operatorname{dim} J_{k, m}^{0}\left(\Gamma_{2}\right)=c(k) \int_{\Gamma_{2} \backslash \mathcal{H}_{2}}(\operatorname{det} Y)^{k-14} \\
\cdot \sum_{M \in \Gamma_{2}} \operatorname{det}\left(\frac{1}{2 i}(Z-\overline{M(Z)})\right)^{4-k} \\
\cdot \overline{j(M, Z)}^{4-k} \operatorname{trace}(\psi(M)) d X d Y,
\end{gathered}
$$

where

$$
c(k)=2^{-13} \pi^{-10} \frac{\Gamma(k-4) \Gamma(k-8)}{\Gamma(k-9) \Gamma(k-13)} .
$$

The leading term in (4.7) is the total contribution from \pm identity of $\Gamma_{2}$, which is given by

$$
I( \pm E)=c(k) \int_{\Gamma_{2} \backslash \mathcal{H}_{2}}(\operatorname{det} Y)^{-10} d X d Y\left(m^{16}+(\text { g.c.d. }(m, 2))^{16}\right)
$$

Of course, the formula (4.7) is still far away an explicit dimension formula for the vector space $J_{k, m}^{0}\left(\Gamma_{2}\right)$ since we only know the contribution from \pm identity. However, we can use it to compute explicitly the contribution from a particular conjugacy class of $\Gamma_{2}$ and hence obtain an approximate formula for $\operatorname{dim} J_{k, m}^{0}\left(\Gamma_{2}\right)$. 


\section{Application to singular modular forms.}

Let $\mathcal{J}_{\mathbb{R}}$ be the set of $3 \times 3$ Hermitian matrices over real Cayley numbers. $\mathcal{J}_{\mathbb{R}}$ consists of matrices of the following form

$(5.1) \quad X=\left[\begin{array}{ccc}\xi_{1} & x_{12} & x_{13} \\ \bar{x}_{12} & \xi_{2} & x_{23} \\ \bar{x}_{13} & \bar{x}_{23} & \xi_{3}\end{array}\right], \quad \xi_{1}, \xi_{2}, \xi_{3} \in \mathbb{R}, x_{12}, x_{13}, x_{23} \in \mathcal{C}_{\mathbb{R}}$

For $X \in \mathcal{J}_{\mathbb{R}}$ as given in (5.1), we define

a) $\operatorname{tr}(X)=\xi_{1}+\xi_{2}+\xi_{3}$,

b)

$\operatorname{det}(X)=\xi_{1} \xi_{2} \xi_{3}-\xi_{1} N\left(x_{23}\right)-\xi_{2} N\left(x_{13}\right)-\xi_{3} N\left(x_{12}\right)+T\left(\left(x_{12} x_{23}\right) \bar{x}_{13}\right)$,

and

c)

$$
\begin{aligned}
X \times X & =X^{2}-\operatorname{tr}(X) X+\frac{1}{2}\left(\operatorname{tr}(X)^{2}-\operatorname{tr}\left(X^{2}\right)\right) E \\
& =\left[\begin{array}{ccc}
\xi_{2} \xi_{3}-N\left(x_{23}\right) & x_{13} \bar{x}_{23}-\xi_{3} x_{12} & x_{12} x_{23}-\xi_{2} x_{13} \\
x_{23} \bar{x}_{13}-\xi_{3} \bar{x}_{12} & \xi_{1} \xi_{3}-N\left(x_{13}\right) & \bar{x}_{12} x_{13}-\xi_{1} x_{23} \\
\bar{x}_{23} \bar{x}_{12}-\xi_{2} \bar{x}_{13} & \bar{x}_{13} x_{12}-\xi_{1} \bar{x}_{23} & \xi_{1} \xi_{2}-N\left(x_{12}\right)
\end{array}\right] .
\end{aligned}
$$

Note that $X$ is invertible if and only if $\operatorname{det} X \neq 0$. In this case the inverse is given by

$$
X^{-1}=\frac{1}{\operatorname{det} X}(X \times X) \text {. }
$$

Also we set

$\operatorname{rank} X=1, \quad$ if and only if $X \neq 0, X \times X=0$,

$\operatorname{rank} X=2, \quad$ if and only if $X \times X \neq 0, \operatorname{det} X=0$, and

$\operatorname{rank} X=3, \quad$ if and only if $\operatorname{det} X \neq 0$.

We supply $\mathcal{J}_{\mathbb{R}}$ with a product defined by

$$
X \circ Y=\frac{1}{2}(X Y+Y X)
$$


where $X Y$ is the ordinary matrix product. Then $\mathcal{J}_{\mathbb{R}}$ becomes a real Jordan algebra with this product. Define an inner product on $\mathcal{J}_{\mathbb{R}}$ by

$$
(X, Y)=\operatorname{tr}(X \circ Y)
$$

Finally, we let $\Re$ be the set of squares $X \circ X$ of elements of $\mathcal{J}_{\mathbb{R}}$ and $\Re^{+}$ the interior of $\Re$. The exceptional domain in $\mathbb{C}^{27}$ is then defined by

$$
\mathcal{H}=\left\{Z=X+i Y: X, Y \in \mathcal{J}_{\mathbb{R}}, Y \in \Re^{+}\right\}
$$

Set $\mathcal{J}_{\mathbf{o}}=\mathcal{J}_{\mathbb{R}} \cap M_{3}(\mathbf{o})$. Here $M_{3}(\mathbf{o})$ is the set of $3 \times 3$ matrices over integral Cayley numbers. For $1 \leq i, j \leq 3$, let $e_{i j}$ be the $3 \times 3$ matrix with 1 at the $i j$-position and 0 elsewhere. When $i \neq j, t \in \mathcal{C}_{\mathbb{R}}$, we let $U_{i j}(t)=E+t e_{i j}, E$ being the $3 \times 3$ identity matrix.

The group of holomorphic automorphisms $\mathcal{G}$ of $\mathcal{H}$ is a Lie group of type $E_{7}$ (see [1]). Let $\Gamma$ be the discrete subgroup of $\mathcal{G}_{\mathbb{R}}$ generated by the following automorphisms of $\mathcal{H}$ :

1) $\iota: Z \longrightarrow-Z^{-1}$,

2) $p_{B}: Z \longrightarrow Z+B, B \in \mathcal{J}_{\mathbf{o}}$, and

3) $t_{U}: Z \longrightarrow Z[U]={ }^{t} \bar{U} Z U, U=U_{i j}(t), t \in \mathbf{o}$.

Let $k$ be an even integer. A holomorphic function $f$ defined on $\mathcal{H}$ is a modular form of weight $k$ with respect to $\Gamma$ if it satisfies the following conditions:

a) $f\left(-Z^{-1}\right)=(\operatorname{det}(-Z))^{k} f(Z)$, and

b) $f(Z[U]+B)=f(Z)$ for all $B \in \mathcal{J}_{\mathbf{o}}$ and $U=U_{i j}(t), t \in \mathbf{o}$.

In particular, from $\mathrm{b}$ ), a modular from $f$ on $\mathcal{H}$ has a Fourier expansion of the form

$$
f(Z)=\sum_{T \in \Re \cap \mathcal{J}_{\mathrm{o}}} a(T) e^{2 \pi i(T, Z)} .
$$

$f$ is a singular modular form if $a(T)=0$ unless $\operatorname{det} T \neq 0$.

Baily (see [1]) considered the Eisenstein series

$$
E_{l}(Z)=\sum_{\gamma \in \Gamma / \Gamma_{0}} \mathbf{j}(\gamma, Z)^{l}, \quad Z \in \mathcal{H}
$$


Here $\Gamma_{0}$ is the subgroup of $\Gamma$ generated by $p_{B}, t_{U}$ with $B \in \mathcal{J}_{\mathbf{o}}, U=$ $U_{i j}(t), t \in \mathbf{o .} \mathbf{j}(\gamma, Z)$ is the determinant of the Jacobian matrix of $\gamma$ at $Z$ and it has the following properties:

1) $\mathbf{j}\left(p_{B}, Z\right)=1$ for all $B \in \mathcal{J}_{\mathbb{R}}$,

2) $\mathbf{j}\left(t_{U}, Z\right)=1$ for all $U=U_{i j}(t), t \in \mathbf{o}$, and

3) $\mathbf{j}(\iota, Z)=(\operatorname{det}(-Z))^{-18}$.

For any positive even integer $l$, the series in (5.5) converges absolutely and uniformly on any compact subset of $\mathcal{H}$. Hence $E_{l}$ is a modular form of weight $18 l$ with respect to $\Gamma$ on $\mathcal{H}$ and has a Fourier expansion

$$
E_{l}(Z)=\sum_{T \in \mathcal{J}_{\mathrm{o}} \cap \Re} a_{l}(T) e^{2 \pi i(T, Z)} .
$$

Baily proved that the Fourier coefficients $a_{l}(T)$ of $E_{l}(Z)$ are rational numbers and concluded that the Satake compactification of $\mathcal{H} / \Gamma$ has a biregularly equivalent projective model defined over the rational number field (see [1]).

In 1993, Kim considered the non-holomorphic Eisenstein series (see [13])

$$
E_{k, s}(Z)=\sum_{\gamma \in \Gamma / \Gamma_{0}} j(\gamma, Z)^{-k}|j(\gamma, Z)|^{-s},
$$

where $k$ is a positive even integer, $s \in \mathbb{C}$ with $k+\operatorname{Re} s>18$, and $j(g, Z)$ is a factor of $\mathbf{j}(g, Z)$ with

$$
\mathbf{j}(g, Z)=j(g, Z)^{-18} .
$$

He proved among other things that as a function of $s, E_{k, s}(Z)$ has a meromorphic analytic continuation in the whole complex plane. Furthermore, $E_{4,0}(Z)$ and $E_{8,0}(Z)$ are singular modular forms of weight 4 and 8 , respectively. Here we shall determine explicitly the Fourier coefficients of these singular modular forms with our knowledge in Jacobi forms of degree one and degree two.

Proposition 11. Let $E_{4}(Z)$ be a modular form of weight 4 on the exceptional domain with the Fourier expansion

$$
E_{4}(Z)=\sum_{T \in \mathcal{J}_{\mathrm{o}} \cap \Re} a(T) e^{2 \pi i(T, Z)} .
$$


Then $a(T)=0$ unless $\operatorname{rank} T \leq 1$. If $a(0)=1$ is given, then for $\operatorname{rank} T=1$

$$
a(T)=240 \sum_{d \mid \varepsilon(T)} d^{3}
$$

where $\varepsilon(T)$ is the largest integer $d$ such that $d^{-1} T \in \mathcal{J}_{\mathbf{o}}$.

ProOF. Let

$$
\varphi_{0}\left(Z_{1}\right)+\sum_{m=1}^{\infty} \varphi_{m}\left(Z_{1}, W\right) e^{2 \pi i m z_{3}}
$$

be the Jacobi-Fourier expansion of $E_{4}(Z)$ with

$$
Z=\left[\begin{array}{cc}
Z_{1} & W \\
{ }^{t} \bar{W} & z_{3}
\end{array}\right]
$$

Then $\varphi_{0}\left(Z_{1}\right)$ is a modular form of weight 4 on $\mathcal{H}_{2}$, hence it is a constant multiple of $f_{4}\left(Z_{1}\right)$ as constructed in [6] since the modular forms of weight 4 has dimension 1 . Note that $a(0)=1$, it follows $\varphi_{0}\left(Z_{1}\right)=$ $f_{4}\left(Z_{1}\right)$ and $a\left(\begin{array}{cc}T_{1} & 0 \\ 0 & 0\end{array}\right)$ is given by $240 \sum_{d \mid \varepsilon\left(T_{1}\right)} d^{3}$ if $\operatorname{det} T_{1}=0, T_{1} \neq 0$.

On the other hand, $\varphi_{m}\left(Z_{1}, W\right)$ is a Jacobi form of weight 4 and index $m$ on $\mathcal{H}_{2} \times \mathcal{C}_{\mathbb{C}}^{2}$. By Proposition 13, we are able to decompose $\varphi_{m}\left(Z_{1}, W\right)$ into

$$
\sum_{\mathbf{q}:(\mathbf{o} / m \mathbf{o})^{2}} F_{\mathbf{q}}\left(Z_{1}\right) \vartheta_{m, \mathbf{q}}\left(Z_{1}, W\right)
$$

with

$$
F_{\mathbf{q}}\left(Z_{1}\right)=\sum_{\substack{T \in \Lambda_{2} \\
T>\mathbf{q}^{t} \overline{\mathbf{q}} / m}} a\left(\begin{array}{cc}
T_{1} & \mathbf{q} \\
{ }^{t} \overline{\mathbf{q}} & m
\end{array}\right) e^{2 \pi i\left(T-\mathbf{q}^{t} \overline{\mathbf{q}} / m, Z_{1}\right)}
$$

By Proposition 4, we know that

$$
F\left(Z_{1}\right)={ }^{t}\left(F_{\mathbf{q}}\left(Z_{1}\right)\right)_{\mathbf{q}:(\mathbf{o} / m \mathbf{o})^{2}}
$$

is a vector-valued modular form of weight 0 . It forces that $F_{\mathbf{q}}\left(Z_{1}\right)$ is a constant and hence

$$
a\left(\begin{array}{cc}
T_{1} & \mathbf{q} \\
t \overline{\mathbf{q}} & m
\end{array}\right)=0
$$

unless $T_{1}=\mathbf{q}^{t} \overline{\mathbf{q}} / m$. This proves $a(T)=0$ unless rank $T \leq 1$. For $T \in \mathcal{J}_{\mathrm{o}}$ with $\operatorname{rank} T=1$, we are able to reduce $T$ to $T_{0}=\operatorname{diag}[\varepsilon(T), 0,0]$ 
by a finite number of operations $T \longrightarrow T[U], U=U_{i j}(t), i \neq j, t \in \mathbf{o}$. Thus

$$
a(T)=a\left(T_{0}\right)=240 \sum_{d \mid \varepsilon(T)} d^{3} .
$$

Next we are going to give a relation among Fourier coefficients of the modular form of weight 4 .

Proposition 12. For each positive integer $m$ and $\mathbf{q}={ }^{t}\left(q_{1}, q_{2}\right) \in \mathbf{o}^{2}$, let

$$
T=\left[\begin{array}{cc}
\mathbf{q}^{t} \overline{\mathbf{q}} / m & \mathbf{q} \\
{ }^{t} \overline{\mathbf{q}} & m
\end{array}\right]
$$

and

$$
G_{m}(\mathbf{q})= \begin{cases}240 \sum_{d \mid \varepsilon(T)} d^{3}, & \text { if } T \in \mathcal{J}_{\mathbf{o}} \\ 0, & \text { otherwise }\end{cases}
$$

Then

$$
G_{m}(\mathbf{q})=\frac{1}{m^{8}} \sum_{\mathbf{p}:(\mathbf{o} / m \mathbf{o})^{2}} e^{2 \pi i\left[\sigma\left(q_{1}, p_{1}\right)+\sigma\left(q_{2}, p_{2}\right)\right] / m} G_{m}(\mathbf{p})
$$

Proof. It follows from the fact that $G={ }^{t}\left(G_{m}(\mathbf{q})\right)_{\mathbf{q}:(\mathbf{o} / m \mathbf{o})^{2}}$ is the vector-valued modular form corresponding to $\varphi_{m}\left(Z_{1}, W\right)$, the $m$-th Jacobi-Fourier coefficient of $E_{4}(Z)$. Thus $G$ must satisfy the condition $B$ of Proposition 4. In particular one has

$$
G=\psi(\iota) G .
$$

This is precisely the identity (5.8) in vector form.

REMARK. Indentity (5.8) was proved in [10] directly from the definition of $G_{m}(\mathbf{q})$ and it implies that

$$
\varphi_{m}\left(Z_{1}, W\right)=\sum_{\mathbf{q}:(\mathbf{o} / m \mathbf{o})^{2}} G_{m}(\mathbf{q}) \vartheta_{m, \mathbf{q}}\left(Z_{1}, W\right), \quad\left(Z_{1}, W\right) \in \mathcal{H}_{2} \times \mathcal{C}_{\mathbb{C}}^{2},
$$

is a Jacobi form of weight 4 and index $m$. With $\varphi_{m}\left(Z_{1}, W\right)$ as the $m$-th coefficient, we are able to define a holomorphic function on the exceptional domain as

$$
E(Z)=f_{4}\left(Z_{1}\right)+\sum_{m=1}^{\infty} \varphi_{m}\left(Z_{1}, W\right) e^{2 \pi i m z_{3}}, \quad Z=\left[\begin{array}{cc}
Z_{1} & W \\
t \bar{W} & z_{3}
\end{array}\right] \in \mathcal{H}
$$


With the theory of Jacobi forms on $\mathbf{H} \times \mathcal{C}_{\mathbb{C}}$ as well as Jacobi forms on $\mathcal{H}_{2} \times \mathcal{C}_{\mathbb{C}}^{2}$, we are able to verify that

$$
E\left(-Z^{-1}\right)=(\operatorname{det} Z)^{4} E(Z) .
$$

See [10] or [15] for the details. Consequently, we provide another way to construct the singular modular form of weight 4 on the exceptional domain.

Note that $E_{4}^{2}(Z)$ is a modular form of weight 8 . Indeed it is a singular modular form of weight 8 and its Fourier coefficiets can be determined explicitly by the following proposition, given in [15].

Propositon 13. Let

$$
E_{4}^{2}(Z)=\sum_{T \in \mathcal{J}_{\mathrm{o}} \cap \Re} b(T) e^{2 \pi i(T, Z)} .
$$

Then $b(T)=0$ unless $\operatorname{rank} T \leq 2$ and

$$
\begin{aligned}
& b\left(\left[\begin{array}{cc}
T_{1} & 0 \\
0 & 0
\end{array}\right]\right) \\
& \quad=\left\{\begin{array}{cc}
1, & \text { if } T_{1}=0, \\
480 \sum_{d \mid \varepsilon\left(T_{1}\right)} d^{7}, & \text { if } \operatorname{det} T_{1}=0, T_{1} \neq 0, \\
240 \cdot 480 \sum_{d \mid \varepsilon\left(T_{1}\right)} d^{7} \sum_{d_{1} \mid \operatorname{det}\left(d^{-1} T_{1}\right)} d_{1}^{3}, & \text { if } \operatorname{det} T_{1} \neq 0 .
\end{array}\right.
\end{aligned}
$$

ProOF. The Fourier coefficient $a(T)$ of $E_{4}(Z)$ has the property that $a(T)=0$ unless rank $T \leq 1$. It follows

$$
b(T)=\sum_{T_{1}+T_{2}=T} a\left(T_{1}\right) a\left(T_{2}\right)
$$

is zero unless $\operatorname{rank} T \leq 2$. Let

$$
\psi_{0}\left(Z_{1}\right)+\sum_{m=1}^{\infty} \psi_{m}\left(Z_{1}, W\right) e^{2 \pi i m z_{3}}
$$

be the Jacobi-Fourier expansion of $E_{4}^{2}(Z)$. Then

$$
\psi_{0}\left(Z_{1}\right)=\sum_{T_{1} \in \Lambda_{2}} b\left(\left[\begin{array}{cc}
T_{1} & 0 \\
0 & 0
\end{array}\right]\right) e^{2 \pi i\left(T_{1}, Z_{1}\right)}=\lim _{\lambda \rightarrow \infty} E_{4}^{2}\left(\left[\begin{array}{cc}
Z_{1} & 0 \\
0 & i \lambda
\end{array}\right]\right)
$$


which is a modular form of wieght 8 and hence it is equal to $\left(f_{4}\left(Z_{1}\right)\right)^{2}$. But $\left(f_{4}\left(Z_{1}\right)\right)^{2}$ is the only modular form of weight 8 which is also in the Maaß space, its coefficients satisfy the Maaß condition. So it suffices to know $b\left(\left[\begin{array}{cc}T_{1} & 0 \\ 0 & 0\end{array}\right]\right)$ with $T_{1}=\left(\begin{array}{cc}n & 0 \\ 0 & 0\end{array}\right)$ or $T_{1}=\left(\begin{array}{cc}n & t \\ \bar{t} & 1\end{array}\right), n-N(t) \neq 0$.

Note that

$$
b\left(\left[\begin{array}{cc}
T_{1} & 0 \\
0 & 0
\end{array}\right]\right)=\#\left\{h_{1}, h_{2} \in \mathbf{o}^{2} \mid h_{2}{ }^{t} \bar{h}_{1}+h_{2}{ }^{t} \overline{h_{2}}=T_{1}\right\} .
$$

For $T_{1}=\left(\begin{array}{ll}n & 0 \\ 0 & 0\end{array}\right)$, we have

$$
b\left(\left[\begin{array}{cc}
T_{1} & 0 \\
0 & 0
\end{array}\right]\right)={ }^{\#}\{a, b \in \mathbf{o} \mid N(a)+N(b)=n\}=480 \sum_{d \mid n} d^{7} .
$$

On the other hand, for $T_{1}=\left[\begin{array}{cc}n & t \\ \bar{t} & 1\end{array}\right]$, we have

$$
b\left(\left[\begin{array}{cc}
T_{1} & 0 \\
0 & 0
\end{array}\right]\right)=240 \cdot 480 \sum_{d \mid(n-N(t))} d^{3} .
$$

\section{References.}

[1] Baily Jr., W. L., An exceptional arithematic group and its Eisenstein series. Ann. of Math. 91 (1970), 512-549.

[2] Baily Jr., W. L., Introductory lectures on automorphic forms. Princeton university Press, 1973.

[3] Coxeter, H. S. M., Integral Cayley numbers. Duke Math. J. 13 (1946), 576-578.

[4] Eichler, M., Zagier, D., The theory of Jacobi forms. Progr. Math. 55 Birkhäuser, 1985.

[5] Eie, M., The Maaß space for Cayley numbers. Math. Z. 207 (1991), 645-655.

[6] Eie, M., Krieg, A., The Maaß space on the half-plane of Cayley numbers of degree two. Math. Z. 210 (1992), 113-128.

[7] Eie, M., Krieg, A., The theory of Jacobi forms over the Cayley numbers. Trans. Amer. Math. Soc. 342 (1994), 793-805. 
[8] Eie, M., Fourier coefficients of Jacobi forms over Cayley numbers. Revista Mat. Iberoamericana 11 (1995), 125-142.

[9] Eie, M., The cohomology group associated with Jacobi cusp forms over Cayley numbers. Amer. J. Math. 120 (1998), 811-826.

[10] Eie, M., Chang, S.-T., An arithmetic property of Fourier coefficients of singular modular forms on the exceptional domain. To appear in Trans. Amer. Math. Soc.

[11] Freitag, E., Singular modular forms and theta relations. Lecture Notes in Math. 1487, Springer-Verlag, 1991.

[12] Karel, M. L., Fourier coefficients of certain Eisenstein series. Ann. of Math. 99 (1974), 176-202.

[13] Kim, H., Exceptional modular form of weight 4 on an exceptional domain contained in $\mathbb{C}^{27}$. Revista Mat. Iberoamericana 9 (1993), 139-200.

[14] Krieg, A., Jacobi forms of several variables and the Maaß space. $J$. Number Theorem 56 (1996), 242-255.

[14] Krieg, A., The singular modular forms on the 27-dimensional exceptional domain. Manuscripta Math. 92 (1997), 361-367.

[16] Ziegler, C., Jacobi forms of higher degree. Abb. Math. Semin. Univ. Hamburg 59 (1989), 191-224.

Recibido: 6 de junio de 1.999

Minking Eie*

Department of Mathematics

National Chung Cheng University

Ming-Hsiung, Chia-Yi

Taiwan, REPUBLIC OF CHINA

mkeie@math.ccu.edu.tw

* This work was supported by Department of Mathematics, National Chung Cheng University and by the National Science Foundation of Taiwan, Republic of China. 